\title{
ON A CERTAIN WEIGHTED PARTITION FUNCTION
}

\section{NELSON A. BRIGHAM}

1. Introduction. In the introduction of the preceding paper we remarked that no one has given an asymptotic formula for the number of partitions into primes. As a step in that direction we now give, under the assumption of the Riemann hypothesis, an asymptotic formula for the summatory function $A(k)$ of a certain weighted partition function $a(k)$ involving primes and powers of primes.

As pointed out in the introduction of the previous paper, the generating function of the type of partition we have in mind is given by

$$
g_{\gamma}(s)=\prod_{n=1}^{\infty} \frac{1}{\left(1-e^{-s n}\right)^{\gamma(n)}}=\sum_{k=0}^{\infty} a_{\gamma}(k) e^{-s k},
$$

where $\gamma(n) \geqq 0, R[s]>0$. Using the Mellin formula for $e^{-s}$ we get from (1)

$$
\log g_{\gamma}(s)=\frac{1}{2 \pi i} \int_{3 / 2-\infty i}^{3 / 2+\infty i}\left\{\frac{\Gamma(z)}{s^{z}} \sum_{n=1}^{\infty} \frac{\gamma(n)}{n^{z}} \sum_{m=1}^{\infty} \frac{1}{m^{z+1}}\right\} d z .
$$

If $\gamma(n)$ has been chosen in such a manner as to yield an "amenable" Dirichlet series, the calculus of residues will give more or less useful information concerning $g_{\gamma}(s)$. For example, if $\gamma(n)=1$, then $a_{\gamma}(k)$ $=p(k)$, and the method leads directly to the usual transformation formula for the generating function of the unrestricted partition function. If we consider the weighted partition function involving primes and powers of primes obtained by choosing $\gamma(n)=\Lambda(n)$, we have

$$
\sum_{n=1}^{\infty} \frac{\gamma(n)}{n^{z}}=\sum_{n=1}^{\infty} \frac{\Lambda(n)}{n^{z}}=-\frac{\zeta^{\prime}(z)}{\zeta(z)},
$$

and the properties of the latter function are well known. For this choice of $\gamma(n)$ we drop subscripts on $a_{\gamma}(k)$ and $g_{\gamma}(s)$ and write

$$
A(k)=\sum_{n \leqq k} a(n) .
$$

If we were to choose $\gamma(n)$ as 1 if $n$ is a prime and 0 otherwise, then $a_{\gamma}(k)$ would be exactly the number of partitions of $k$ into primes, but we would have to deal with the less tractable function $\sum p^{-z}$.

Using (2) with $\gamma(n)=\Lambda(n)$, we obtain by means of the calculus of residues an asymptotic formula for $g(s)$ as $s$ tends to $O$ within an

Received by the editors December 8, 1948. 
angle of the form $|\arg s| \leqq \theta_{0}<\pi / 2$, namely

$$
g(s)=K_{2} s^{\log 2 \pi} \exp \left(\frac{\pi^{2}}{6 s}+Z(s)+O(|s|)\right),
$$

where

$$
K_{2}=\exp \left(-1+\frac{\pi^{2}}{24}+\sum_{p} \frac{1}{\rho^{2}}\right)
$$

and

$$
Z(s)=\sum_{\rho} \frac{\pi(2 \pi)^{\rho} \zeta(-\rho)}{\rho s^{\rho} \sin (\pi \rho / 2)}=-\sum_{\rho} \frac{\zeta(1+\rho) \Gamma(\rho)}{s^{\rho}},
$$

the summations being taken over the complex zeros of $\zeta(z)$. We shall show that $Z(s)=o\left(|s|^{-1}\right)$, so that $\pi^{2} / 6 s$ is the dominant term in the exponent of $e$ in (3). Now if we assume the Riemann hypothesis, we can show that $g(s)$ satisfies the conditions of a Tauberian theorem of Ingham [2]. Applying this theorem, we get

$$
A(k) \sim K_{1} \sigma^{1 / 2+\log 2 \pi} \exp \left(\frac{1}{\sigma}\left\{\frac{\pi^{2}}{3}+\sigma Z(\sigma)-\sigma^{2} Z^{\prime}(\sigma)\right\}\right),
$$

where $K_{1}=(3 / 2 \pi)^{1 / 2} K_{2} / \pi$, and $\sigma$ is the positive solution, existent and unique for $k$ sufficiently large, of

$$
\frac{1}{\sigma^{2}}\left(\frac{\pi^{2}}{6}-\sigma^{2} Z^{\prime}(\sigma)\right)=k \text {. }
$$

As $k$ tends to infinity, $\sigma$ tends to 0 and

$$
\sigma Z(\sigma) \rightarrow 0, \quad \sigma^{2} Z^{\prime}(\sigma) \rightarrow 0, \quad \frac{1}{\sigma} \sim \frac{(6 k)^{1 / 2}}{\pi},
$$

in conformity with (9) of the previous paper. In contrast to (9) of the previous paper, the powers of primes make an essential contribution to (6).

In order to get an asymptotic formula for $a(k)$ we would need to apply Ingham's theorem to

$$
\sum_{k=0}^{\infty}\{a(k)-a(k-1)\} e^{-s k}=\left(1-e^{-s}\right) g(s) .
$$

However, the use of Ingham's theorem would require that we know that $a(k)$ is nondecreasing for large $k$, which seems difficult to establish (if true). 
In the Hardy-Ramanujan Farey-arc method [1] ${ }^{1}$, one would consider

$$
h(x)=\prod_{n=1}^{\infty} \frac{1}{\left(1-x^{n}\right)^{\Delta(n)}}
$$

in the neighborhood of its singularities at the points $e^{2 \times a i / b}$, where $a$ and $b$ are integers. In our case we have $x=e^{-s}, R[s]>0$, and consider the approach of $s$ to zero. This corresponds to considering only the most important of the singularities, namely that at $x=1$.

2. The asymptotic formula for $g(s)$. Taking the logarithm of (1), with $\gamma(n)=\Lambda(n)$, we have

$$
\log g(s)=\sum_{n=1}^{\infty} \sum_{m=1}^{\infty} \frac{\Lambda(n)}{m} e^{-s n m},
$$

where $s$ is complex, $|s| \leqq 1,|\arg s| \leqq \theta_{0}<\pi / 2$, and $\log g(1)$ is real.

Using the Mellin transform,

$$
e^{-\bullet}=\frac{1}{2 \pi i} \int_{3 / 2-\infty i}^{3 / 2+\infty i} \frac{\Gamma(z)}{s^{z}} d z,
$$

we find

$$
\log g(s)=\frac{1}{2 \pi i} \int_{3 / 2-\infty i}^{3 / 2+\infty i} \frac{\Gamma(z)}{s^{z}} \sum_{n=1}^{\infty} \frac{\Lambda(n)}{n^{z}} \sum_{m=1}^{\infty} \frac{1}{m^{z+1}} d z,
$$

or

$$
\log g(s)=\frac{-1}{2 \pi i} \int_{3 / 2-\infty i}^{3 / 2+\infty i} \frac{\Gamma(z) \zeta(z+1)}{s^{z}} \frac{\zeta^{\prime}(z)}{\zeta(z)} d z .
$$

Now we consider

$$
\frac{-1}{2 \pi i} \int \frac{\Gamma(z) \zeta(z+1)}{s^{z}} \frac{\zeta^{\prime}(z)}{\zeta(z)} d z
$$

where the integral is taken around the rectangle $3 / 2-T_{0} i, 3 / 2+T_{0} i$, $-3 / 2+T_{0} i,-3 / 2-T_{0} i_{.} T_{0}[3, \mathrm{pp} .340-341]$ is an ordinate between the positive integers $g$ and $g+1$ upon which $\zeta(z)$ has no zeros, and for which

$$
\left|\frac{\zeta^{\prime}\left(x \pm T_{\theta} i\right)}{\zeta\left(x \pm T_{\theta} i\right)}\right|<C \log ^{2} T_{\theta}, \quad-\frac{3}{2} \leqq x \leqq \frac{3}{2}, \quad g \geqq 2 .
$$

1 Numbers in brackets refer to the references cited at the end of the paper. 
Note: We put $z=x+y i$, since we later wish to follow Ingham [2], who uses $s=\sigma+t i$. We then put $y=T_{0}$ for the particular set of ordinates in question, following Landau [3].

We estimate the integral over the end segments $\left(3 / 2+T_{0} i\right.$, $\left.-3 / 2+T_{o} i\right)$ and $\left(-3 / 2-T_{o} i, 3 / 2-T_{0} i\right)$. On these segments we have, in addition to (10), $|\zeta(z+1)| \leqq C T_{0}^{2}[5$, p. 276]. For fixed $x$, and $y \rightarrow \pm \infty$, it is easy to show that (cf. [4, Satz 299])

$$
|\Gamma(z)| \leqq C e^{-x T_{\theta} / 2} T_{g} \text {. }
$$

Putting ( $s$ fixed)

$$
s=r e^{i \theta}, \quad 0<r \leqq 1, \quad|\theta| \leqq \theta_{0}<\pi / 2,
$$

we have $\left|s^{-z}\right| \leqq C e^{T \theta_{0} 0}$.

Thus the absolute value of the integral (9) over the end segments is less than or equal to

$$
C\left(e^{-\pi T_{0} / 2} T_{\theta}\right)\left(T_{\theta}^{2}\right)\left(e^{T_{\theta} \theta_{0}}\right)\left(\log ^{2} T_{\theta}\right) \leqq C \exp \left(T_{\theta}\left(\theta_{0}-\pi / 2\right)+4 \log T_{\theta}\right) .
$$

With $\pi / 2-\theta_{0}=\theta_{1}>0$, we may absorb the $4 \log T_{0}$ by using as an exponent $\theta_{1} T_{0} / 2$. We obtain as our final estimation,

$$
C e^{-\theta_{1} T_{\theta} / 2}, \quad \theta_{1}>0, \quad T_{0}>2 .
$$

If we denote the sum of the residues within the rectangle of integration by $\Sigma_{T_{\theta}}$, (9) becomes

$$
\begin{aligned}
& \frac{-1}{2 \pi i} \int_{3 / 2-T_{\theta} i}^{3 / 2+T_{\theta} i} \frac{\Gamma(z) \zeta(z+1)}{s^{z}} \frac{\zeta^{\prime}(z)}{\zeta(z)} d z \\
& \quad+\frac{-1}{2 \pi i} \int_{-3 / 2+T_{\theta} i}^{-3 / 2-T_{\theta} i} \frac{\Gamma(z) \zeta(z+1)}{s^{z}} \frac{\zeta^{\prime}(z)}{\zeta(z)} d z+O\left(e^{-\theta_{1} T_{\theta} / 2}\right)=\Sigma_{T_{\theta}} .
\end{aligned}
$$

Next we compute $\Sigma_{T_{0}}$. For this purpose we alter the integrand of (12) by means of

$$
\zeta(1+z)=2(2 \pi) z(-z) \cos \frac{\pi z}{2} \Gamma(-z) \quad[3, \text { p. 285] }
$$

and

$$
\Gamma(z) \Gamma(-z)=\frac{-\pi}{z \sin \pi z}
$$

which gives

$$
\frac{\pi(2 \pi)^{z}}{z \sin \pi z / 2} \cdot \frac{1}{s^{z}} \cdot \frac{\zeta^{\prime}(z)}{\zeta(z)} \cdot \zeta(-z) .
$$


At $z=1, z=-1$, and at each of the complex zeros of $\zeta(z),(15)$ has a simple pole. (15) has a double pole at the origin.

We compute the residues at the simple poles first.

Since

$$
\frac{\zeta^{\prime}(z)}{\zeta(z)}=-\frac{1}{z-1}+\cdots,
$$

the residue at $z=1$ is

$$
-\pi(2 \pi) \frac{1}{s} \zeta(-1)=\frac{\pi^{2}}{6 s} .
$$

The sum of the residues at the complex zeros of $\zeta(z)$ is

$$
\sum_{\rho,|\gamma|<T_{\theta}} \frac{\pi(2 \pi) \rho \zeta(-\rho)}{\rho\left(\sin (\pi \rho / 2) s^{\rho}\right.},
$$

where $\rho=\beta+\gamma i$ runs over the complex zeros of $\zeta(z)$. Each zero is counted to its multiplicity.

Since $\zeta(z)=1 /(z-1)+\cdots$, the residue at $z=-1$ is

$$
-\frac{1}{2} s \frac{\zeta^{\prime}(-1)}{\zeta(-1)}=O(r), \quad r \rightarrow 0 .
$$

In order to obtain the residue at the double pole at the origin, we use the following expansions $(P(z)$ denotes a power series convergent for $|z|$ small enough)

$$
\begin{aligned}
\left(\frac{2 \pi}{s}\right)^{z}= & 1+z \log \frac{2 \pi}{s}+z^{2} P(z), \\
\frac{1}{\sin (\pi z / 2)}= & \frac{2}{\pi z}+z P(z), \\
\zeta(z)= & -\frac{1}{2}-\frac{1}{2}(\log 2 \pi) z \\
& -\frac{1}{4}\left(1-\frac{\pi^{2}}{24}+\log ^{2} 2 \pi-\sum_{\rho} \frac{1}{\rho^{2}}\right) z^{2}+z^{3} P(z), \\
\zeta^{\prime}(z)= & -\frac{1}{2} \log 2 \pi \\
& -\frac{1}{2}\left(1-\frac{\pi^{2}}{24}+\log ^{2} 2 \pi-\sum_{\rho} \frac{1}{\rho^{2}}\right) z+z^{2} P(z),
\end{aligned}
$$




$$
\frac{1}{\zeta(z)}=-2+2(\log 2 \pi) z+z^{2} P(z) .
$$

The expansions for $\zeta(z)$ come from

$$
\zeta(z)=\frac{e^{b z}}{2(z-1) \Gamma(z / 2+1)} \prod_{p}\left\{\left(1-\frac{z}{\rho}\right) e^{z / \rho}\right\}[3, \text { p. 115] }
$$

and

$$
\frac{1}{\Gamma(z+1)}=e^{\gamma z} \prod_{n=1}^{\infty}\left\{\left(1+\frac{z}{n}\right) e^{-z / n}\right\},
$$

where $b=\log 2 \pi-1-\gamma / 2, \gamma$ being Euler's constant.

We find that the residue at $z=0$ is

$$
\log 2 \pi \log s-1+\frac{\pi^{2}}{24}+\sum_{\rho} \frac{1}{\rho^{2}} .
$$

Inserting (16), (17), (18), and (19) in (12), and allowing $T_{0} \rightarrow \infty$, we obtain from (8)

$$
\begin{aligned}
\log g(s)= & \frac{1}{2 \pi i} \int_{-3 / 2+\infty i}^{-3 / 2-\infty i} \frac{\Gamma(z) \zeta(z+1)}{s^{z}} \frac{\zeta^{\prime}(z)}{\zeta(z)} d z+\frac{\pi^{2}}{6 s}+Z(s) \\
& +\log 2 \pi \log s-1+\frac{\pi^{2}}{24}+\sum_{\rho} \frac{1}{\rho^{2}}+O(r),
\end{aligned}
$$

where $Z(s)$ is given by (5).

In the integrand of the absolutely convergent integral which appears in (20), (11) gives $\left|s^{-z}\right|=r^{3 / 2} e^{-\theta y}$. Thus the integral is $O\left(r^{8 / 2}\right)=O(r)$ for $r$ tending to zero, and so we get the transformation formula

(21) $\log g(s)=\frac{\pi^{2}}{6 s}+Z(s)+\log 2 \pi \log s-1+\frac{\pi^{2}}{24}+\sum_{\rho} \frac{1}{\rho^{2}}+O(r)$.

This gives (3) at once.

Since $1 /\left(1-e^{-s n}\right)=(1 / s n)\{1+o(1)\}$ as $|s| \rightarrow 0$, each factor of (1) (with $\gamma(n)=\Lambda(n)$ ) for which $\Lambda(n) \neq 0$ makes a contribution of importance to (3). Thus the powers of primes make an essential contribution to the transformation formula, and hence ultimately to our result for $A(k)$. The "size" of this contribution is readily seen: Write

$$
g(s)=g_{1}(s) g_{2}(s)=\left(\prod_{p} \frac{1}{\left(1-\sigma^{s p}\right)^{\log p}}\right)\left(\prod_{p^{m}, m \geq 2} \frac{1}{\left(1-e^{-s p m}\right)^{\log p}}\right),
$$


and since $\sum_{p^{m} \leqq x ; m \geq 2} \log p \sim x^{1 / 2},(6)$ of the preceding paper applied to $g_{1}(s)$ and $g_{2}(s)$ separately gives

$$
g(s)=\exp \left(\frac{\pi^{2}}{6 s}\{1+o(1)\}+\frac{\pi^{1 / 2}}{4} \zeta\left(\frac{3}{2}\right) \frac{1}{s^{1 / 2}}\{1+o(1)\}\right) .
$$

Comparison of (21) and (22) shows that $\lim _{r \rightarrow 0} r Z(r)=0$. For future use we prove more, namely

$$
\lim _{r \rightarrow 0} r^{n+1} Z^{(n)}(s)=0,
$$

where $Z^{(n)}(s)$ means the $n$th derivative, $n \geqq 0$.

Using the first form of $Z$ given in (5), we have, with $\rho=\beta+\gamma i$, $r^{n+1}\left|Z^{(n)}(s)\right| \leqq C r^{n+1} \sum_{\rho} \frac{|\zeta(-\rho)| \cdot|(\rho)(\rho+1) \cdots(\rho+n-1)|}{|\rho| \cdot\left|s^{\rho+n}\right| \cdot|\sin \pi \rho / 2|} \cdot$

But

$$
\frac{|\zeta(-\rho)| \cdot|\rho(\rho+1) \cdots(\rho+n-1)|}{|\rho|} \leqq C \gamma^{2} \gamma^{n-1}=C \gamma^{n+1}[5, \text { p. 276] }
$$

and

$$
\frac{1}{\left|s^{\rho+n}\right|}=\frac{1}{r^{n}} \frac{1}{\left|s^{\rho}\right|} \leqq \frac{C}{r^{n}} \frac{e^{\theta_{0}|r|}}{r^{\beta}}
$$

while

$$
\frac{1}{|\sin (\pi \rho / 2)|} \leqq C e^{-\pi|\gamma| / 2}
$$

Using $\pi / 2-\theta_{0}=\theta_{1}$ as before, we have

$$
r^{n+1}\left|Z^{(n)}(s)\right| \leqq C r \sum_{\rho} \frac{e^{-\theta_{1}|\gamma| / 2}}{r^{\beta}} .
$$

But

$$
\beta \leqq 1-\frac{a}{\log |\gamma|}, \quad a>0 \quad[3, \text { p. 321] }
$$

and so

$$
r^{n+1}\left|Z^{(n)}(s)\right| \leqq C r \sum_{|\gamma| \leq T} \frac{e^{-0_{1}|\gamma| / 2}}{r^{1-\alpha / \log |\gamma|}}+C \sum_{|\gamma|>T} e^{-\theta_{1}|\gamma| / 2}
$$

2 The second factor of the numerator is to be taken as 1 in case $n$ is zero. 
It is well known that the number of complex zeros of $\zeta(z)$ with imaginary part between $T$ and $T+1$ is $O(\log T)$ and the number with imaginary part less than or equal to $T$ is $O(T \log T)[3, \mathrm{pp} .337-$ 338]. Hence

$$
\begin{aligned}
& r^{n+1}\left|Z^{(n)}(s)\right| \\
& \leqq C(T \log T) \boldsymbol{r}^{a / \log T}+C \sum_{n=T}^{\infty} e^{-\theta_{1} n / 4} \\
& \leqq C \exp \left(\frac{\log ^{2} T+\log T \log \log T+a \log r}{\log T}\right)+C e^{-\theta_{1} T / 4} .
\end{aligned}
$$

Put $T=-\log r$ and both terms $\rightarrow 0$ as $r \rightarrow 0$. This establishes (23).

3. Application of Ingham's Tauberian Theorem. We see at once that

$$
g(s)=\int_{0}^{\infty} e^{-u s} d A(u)=s \int_{0}^{\infty} e^{-u s} A(u) d u,
$$

where $A(u)=A(k)$ for $k \leqq u<k+1$, and we now are in a position to apply Ingham's theorem [2]. Since it is an integral part of what follows, we quote the relevant portions of this theorem in their entirety. All statements apply for $\sigma$ sufficiently small (or $\omega$ sufficiently large). Open segments: $(a, b)$; closed segments: $[a, b]$.

We quote: "Let there be given two functions $\phi(s)$ and $\chi(s)$, and a domain $D$ of the $s$-plane, satisfying the following conditions:

a.) $\phi(s)$ and $\chi(s)$ are regular in $D$, and real and positive on a segment $(0, h]$ of the positive real axis lying in $D$;

b.) $-\sigma \phi^{\prime}(\sigma)$ tends monotonically to infinity as $\sigma$ tends to $0 \cdots$;

c.) $\frac{\delta(\sigma) / \sigma}{\left\{\phi^{\prime \prime}(\sigma)\right\}^{1 / 2} /-\phi^{\prime}(\sigma)}$ tends to $\infty$ as $\sigma$ tends to 0 ,

where $\delta(\sigma)$ is the distance of the point $\sigma$ from the complement of $D \cdots$;

d.) $\phi^{\prime \prime}(\sigma+z)=O\left\{\phi^{\prime \prime}(\sigma)\right\}$,

$$
\chi(\sigma+z)=O\{\chi(\sigma)\}
$$

uniformly for $|z|<\delta(\sigma)$ when $\sigma$ tends monotonically to 0 .

Define

$$
f_{0}(s)=\chi(s) e^{\phi(0)}, \quad F_{0}(s)=\frac{f_{0}(s)}{s},
$$




$$
A_{0}(\omega)=\frac{\chi(\sigma) e^{\phi(\sigma)+\omega \sigma}}{\left\{2 \pi \sigma^{2} \phi^{\prime \prime}(\sigma)\right\}^{1 / 2}}=\frac{F_{0}(\sigma) e^{\omega \sigma}}{\left\{2 \pi \phi^{\prime \prime}(\sigma)\right\}^{1 / 2}},
$$

where $\sigma=\sigma_{\omega}$ is the solution [existent and unique for sufficiently large $\omega$, by (a.) and (b.)] of

$$
-\phi^{\prime}(\sigma)=\omega \quad(0<\sigma \leqq h) .
$$

In these circumstances we have: Suppose that the integral

$$
f(s)=\int_{0}^{\infty} e^{-u s} d A(u), \quad A(0)=0, \quad(s=\sigma+i t)
$$

is convergent for $\sigma>0$, and that

(i.) $f(s) \sim f_{0}(s)$ when $s \rightarrow 0$ in $D$;

(ii.) $f(s)=O\left\{f_{0}(|s|)\right\}$ when $s \rightarrow 0$ in some fixed angle ' $\Delta$ ' of the form $|t| \leqq \Delta \sigma,(0<\Delta<\infty)$;

(iii.) $A(u)$ is increasing (in the wide sense) for $u \geqq 0$.

If (ii) holds for every fixed $\Delta$, then

$$
A(u) \sim A_{0}(u) \text { as } u \rightarrow \infty . "
$$

We note that Ingham's theorem has $A(0)=0$, whereas it has been convenient for us to write $A(0)=1$. This does not affect the result.

We desire to apply Ingham's theorem with

$$
\chi(s)=K_{2} s^{\log 2 \pi}
$$

and

$$
\phi(s)=\frac{\pi^{2}}{6 s}+Z(s) .
$$

It must therefore be established that the conditions of Ingham's theorem are satisfied by our functions.

For $D$ we choose the region bounded by the rays $|\theta|=\pi / 4$ and the arc $|s|=1 . \delta(\sigma)$ becomes merely the distance from $\sigma$ to the boundary of $D$. From a figure we see that:

$$
\delta(\sigma)=2^{1 / 2} \sigma / 2, \quad \sigma \leqq 1 / 2 .
$$

Hypothesis (a):

$\phi(s)$ and $\chi(s)$ are regular in $D$.

$\chi(s)$ is real and positive on the segment $(0,1]$.

In virtue of (23) and the fact that the complex zeros of $\zeta(z)$ occur in conjugate pairs, $\phi(s)$ is also real and positive on a segment $(0, h]$ of the positive real axis for $h$ sufficiently small. 
Hypothesis (b):

$$
\begin{aligned}
-\sigma \phi^{\prime}(\sigma) & =\frac{\pi^{2}}{6 \sigma}-\sigma Z^{\prime}(\sigma), \\
\left(-\sigma \phi^{\prime}(\sigma)\right)^{\prime} & =\frac{-\pi^{2} / 6-\sigma^{2} Z^{\prime}(\sigma)-\sigma^{2} Z^{\prime \prime}(\sigma)}{\sigma^{2}} .
\end{aligned}
$$

Using (23), we find that the slope of $-\sigma \phi^{\prime}(\sigma)$ is negative for sufficiently small $\sigma$. Hence $-\sigma \phi^{\prime}(\sigma)$ tends monotonically to $\infty$ as $\sigma \rightarrow 0$ in the interval $(0, h]$ for sufficiently small $h$.

Hypothesis (c): $\delta(\sigma) / \sigma=2^{1 / 2} / 2$ while

$$
\frac{\left\{\phi^{\prime \prime}(\sigma)\right\}^{1 / 2}}{-\phi^{\prime}(\sigma)}=\sigma^{1 / 2} \frac{\left\{\pi^{2} / 3+\sigma^{8} Z^{\prime \prime}(\sigma)\right\}^{1 / 2}}{\pi^{2} / 6-\sigma^{2} Z^{\prime}(\sigma)} \rightarrow 0
$$

as $\sigma \rightarrow 0$ by (23).

Hypothesis (d): We see from a figure that $\sigma+z$ lies within a circle whose center is $\sigma$ and whose radius is $\delta(\sigma)=\left((2)^{1 / 2} / 2\right) \sigma, \sigma<1 / 2$. Thus,

$$
\left(1-\frac{(2)^{1 / 2}}{2}\right) \sigma<|\sigma+z|<\left(1+\frac{(2)^{1 / 2}}{2}\right) \sigma(\sigma \text { small enough }) .
$$

So

$$
|\chi(\sigma+z)| \leqq C \sigma^{\log 2 \pi} \leqq C \chi(\sigma) \quad \text { uniformly in } z .
$$

Putting the first inequality of (24) into $\left|\phi^{\prime \prime}(\sigma+z)\right|$ we have, from (23),

$$
\left|\phi^{\prime \prime}(\sigma+z)\right| \leqq C / \sigma^{3} \text {. }
$$

On the other hand, using (23) again, we obtain for sufficiently small $\sigma$

$$
\phi^{\prime \prime}(\sigma)>\pi^{2} / 4 \sigma^{3} \text {. }
$$

Thus: $\left|\phi^{\prime \prime}(\sigma+z)\right|<c \phi^{\prime \prime}(\sigma)$ uniformly in $z$.

Hypothesis (i): This has already been established. See (21).

Hypothesis (ii): We must show that

$$
|g(s)| \leqq C \chi(r) e^{\phi(r)},
$$

$s \rightarrow 0$, for every fixed $\theta_{0}$ of (11). This is equivalent to proving that

$$
\left|s^{\log 2 \pi} \exp \left(\frac{\pi^{2}}{6 s}+Z(s)\right)\right| \leqq r^{\log 2 \pi} \exp \left(\frac{\pi^{2}}{6 r}+Z(r)+C\right) .
$$

The problem thus reduces to finding a $C$, independent of $s$, such that: 


$$
R\left[\frac{\pi^{2}}{6 s}+Z(s)\right] \leqq \frac{\pi^{2}}{6 r}+R[Z(r)]+C .
$$

Clearly, $R\left[\pi^{2} / 6 s\right]=\pi^{2} \cos \theta / 6 r$, see (11).

Now we analyze:

$$
Z(s)=-\sum_{\rho} \frac{\zeta(1+\rho) \Gamma(\rho)}{s^{\rho}} .
$$

In the first place,

$$
s^{-\rho}=\left(r e^{i \theta}\right)^{-\beta-\gamma i}=\frac{e^{\gamma \theta}}{\gamma^{\beta}}\{\cos (\beta \theta+\gamma \log \gamma)-i \sin (\beta \theta+\gamma \log r)\} .
$$

Put

$$
-\zeta(1+\rho) \Gamma(\rho)=X(\rho)+i Y(\rho)=X+Y i
$$

This gives

$$
R[Z(s)]=\sum_{\rho} \frac{1}{r^{\beta}}\left\{X e^{r 0} \cos (\theta \beta+\gamma \log r)+Y e^{r \theta} \sin (\theta \beta+\gamma \log r)\right\} .
$$

Thus we must show that

$$
\begin{aligned}
\frac{\pi^{2}}{6 r}(1-\cos \theta)+C \geqq & \sum_{\rho} \frac{1}{r^{\beta}}\left\{X e^{\gamma \theta} \cos (\theta \beta+\gamma \log r)\right. \\
& +Y e^{\gamma \theta} \sin (\theta \beta+\gamma \log r) \\
& -X \cos (\gamma \log r)-Y \sin (\gamma \log r)\} .
\end{aligned}
$$

Now $1-\cos \theta \geqq \theta^{2} / 4$ for $|\theta| \leqq \pi / 2$. If we expand $\cos (\theta \beta+\gamma \log r)$ and $\sin (\theta \beta+\gamma \log \gamma)$, and collect terms, it will be sufficient to show that:

$$
\begin{aligned}
\frac{\pi^{2} \theta^{2}}{24 r}+C \geqq & \sum_{\dot{\rho}} \frac{1}{\gamma^{\beta}}\left\{[X \cos (\gamma \log \gamma)+Y \sin (\gamma \log r)] \cdot\left[e^{\gamma \theta} \cos \theta \beta-1\right]\right. \\
& \left.+[Y \cos (\gamma \log \gamma)-X \sin (\gamma \log r)]\left[e^{\gamma \theta} \sin \theta \beta\right]\right\} .
\end{aligned}
$$

But $e^{\gamma \theta} \cos \theta \beta-1=\left(e^{\gamma 0}-1\right) \cos \theta \beta+(\cos \theta \beta-1)$, and for all real $x$, $\left|e^{x}-1\right| \leqq|x| e^{|x|}$. Also

$$
|\cos \theta \beta-1|=2 \sin ^{2} \frac{\theta \beta}{2} \leqq \frac{\theta^{2} \beta^{2}}{2} \leqq \frac{|\theta| \pi}{4} .
$$

Thus $\left|e^{\gamma \theta} \cos \theta \beta-1\right| \leqq|\gamma \theta| e^{\mid \gamma \theta 1}+\pi|\theta| / 4$, while $|\sin \theta \beta| \leqq|\theta| \beta \leqq|\theta|$. Hence it will be sufficient to show that 


$$
\begin{aligned}
\frac{\pi^{2} \theta^{2}}{24 r}+C \geqq \sum_{\rho} \frac{1}{r^{\beta}}\left\{(|X|+|Y|)\left(|\gamma \theta| e^{|\gamma| \theta_{0}}+\frac{\pi|\theta|}{4}\right)\right. & \\
& \left.+|\theta| e^{|\gamma| 0_{0}}(|X|+|Y|)\right\} .
\end{aligned}
$$

Or, with all $\beta \leqq \beta_{0}, 1 / 2 \leqq \beta_{0} \leqq 1$, since $|\gamma|>2$ for all $\gamma$,

$$
\frac{\pi^{2} \theta^{2}}{24 r}+C \geqq \frac{|\theta|}{r^{\beta_{0}}} \sum_{\rho} 2|\gamma|(|X|+|Y|) e^{|\gamma| \theta_{0}} .
$$

Referring to the definition of $X$ and $Y$ in (25), we see that the above series converges for all fixed values of $\theta_{0}, \theta_{0}<\pi / 2$. The problem finally reduces, then, to finding a $C_{1}$ such that

$$
\frac{\theta^{2}}{r}+C_{1} \geqq \frac{2 K_{3}|\theta|}{r^{\beta_{0}}} \quad \text { where } K_{3} \text { is a numerical constant. }
$$

This is equivalent to

$$
\left(\frac{|\theta|}{r^{1 / 2}}-K_{3}\right)^{2} \geqq 0 \quad \text { with } \beta_{0}=\frac{1}{2} \text { and } C_{1}=K_{8}^{2} .
$$

The full Riemann hypothesis is essential for our method, since (26) can be written

$$
\frac{1}{r^{2 \beta_{0}-1}}\left(\frac{|\theta|}{r^{1-\beta_{0}}}-K_{3}\right)^{2}+C_{1}-\frac{K_{3}^{2}}{r^{2 \beta_{0}-1}} \geqq 0 .
$$

Unless $2 \beta_{0}-1=0$, no $C_{1}$ can be found which will satisfy (27) for all values of $s$ simultaneously. For if $\beta_{0}=1$, we could choose $\theta=r^{1 / 2}$, and then find the contradiction that $\left(K_{3}^{2}-\left(K_{3}-r^{1 / 2}\right)^{2}\right) / r$ is bounded. If $1 / 2<\beta_{0}<1$, we could choose $\theta$ as $K_{3} r^{1-\beta_{0}}$ for sufficiently small $r$, and then we would have the contradiction that $K_{3}^{2} / r^{2 \beta_{0}-1}$ is bounded.

Hypothesis (iii): This is assured by the definition of $A(u)$.

Since the conditions of Ingham's theorem are satisfied, we have

$$
A(k) \sim \frac{K_{2} \sigma^{\log 2 \pi} \exp \left(\pi^{2} / 6 \sigma+Z(\sigma)+k \sigma\right)}{\left\{2 \pi \sigma^{2}\left(\pi^{2} / 3 \sigma^{3}+Z^{\prime \prime}(\sigma)\right)\right\}^{1 / 2}},
$$

where (7) holds.

Because of (23), the actual contribution of the denominator is

$$
\left((2 \pi)^{1 / 2} \sigma \frac{\pi}{(3)^{1 / 2} \sigma^{8 / 2}}\right)^{-1}\left(1+\frac{3 \sigma^{3}}{\pi^{2}} Z^{\prime \prime}(\sigma)\right)^{-1 / 2} \sim \frac{(3)^{1 / 2}}{(2)^{1 / 2}} \frac{\sigma^{1 / 2}}{\pi^{3 / 2}} .
$$


Substituting (7) and (29) into (28), we obtain our final result, (6), where

$$
K_{1}=\frac{(3)^{1 / 2}}{(2)^{1 / 2} \pi^{3 / 2}} K_{2}=\frac{(6)^{1 / 2}}{2 \pi^{3 / 2}} \exp \left(-1+\frac{\pi^{2}}{24}+\sum_{p} \frac{1}{\rho^{2}}\right),
$$

and where $\sigma$ is the solution, existent and unique for $\sigma$ small enough, of (7). $Z(s)$ is given by (5).

The author wishes to express his gratitude to Professor Hans A. Rademacher of the University of Pennsylvania for suggesting this problem and for his kind assistance and constant encouragement. This paper is presented in partial fulfillment of the requirements for the degree of Doctor of Philosophy at the University of Pennsylvania.

\section{REFERENCES}

1. G. H. Hardy and S. Ramanujan, Asymptotic formulae in combinatory analysis, Proc. London Math. Soc. vol. 17 (1918) pp. 75-115. Also in Collected Papers of Srinivasa Ramanujan.

2. A. E. Ingham, $A$ tauberian theorem for partitions, Ann. of Math. vol. 42 (1941) pp. 1075-1090.

3. E. Landau, Handbuch der Lehre von der Verteilung der Primzahlen, vol. 1.

4. - Vorlesungen viber Zahlentheorie, vol. 1.

5. E. T. Whittaker and G. N. Watson, Modern analysis.

University of Pennsylvania 